\title{
Dangerous liaisons?
}

I sometimes feel that I am so dominated by circumstances and coincidences that I have little free choice, for example, when approaching an editorial. A case in point was a few days last month during which I attended a well-sponsored meeting of the Ontario Lung Association, reviewed a couple of papers reporting drug trials, read of the threats of litigation made by pharmaceutical companies to two Ontario researchers, heard of a public apology made by the New England Journal of Medicine regarding reviewers' conflicts of interest and received a critical letter from Dr Rob McFadden, an associate editor of the Canadian Respiratory Journal, about a sponsored publication that accompanied the last issue of 1999. All this I suppose reflects our rather ambivalent relationship with the pharmaceutical industry that supports many professional and academic programs but clearly expects some returns in addition to corporate tax benefits. We are now dependent on the industry's financial backing for academic and professional meetings that have become so large that they require large and expensive venues. But then the industry has the right to expect some return on its 'investment' in such meetings. Nowhere was this more apparent than at the annual meeting of the Royal College in Montreal, where the pathetically small attendance was matched by the apathy of the pharmaceutical exhibition; the most popular exhibit was that of Jaguar cars offering free test drives of their new model. With other academic programs we are also in debt to drug firms who provide funds for visiting speakers, refreshments and small meetings, which in 'the good old days' seemed easy to come by from local educational grants; such funds are a boost to morale, even if their academic purpose may seem a little strained at times. The annual competition for residents and fellows, held last year in Ste Adèle, Quebec (published in Can Respir J 2000;7:84-94) was a good example where legitimate academic and social objectives were amply met. Other meetings seem to follow less altruistic principles, such as those organized in an attractive part of the world to present and discuss new drug developments; the papers presented usually describe work that has been driven by the company rather than by the researcher, who may use an inflated budget to 'piggy-back' the research he or she really wants to do. The resulting papers that are pulled together in an unpeer-reviewed publication may leave a lot to be desired, which was Dr McFadden's point.

Journals seem to have had a number of problems related to pharmaceutical-sponsored research papers, and have been striving to develop an approach to the ethics of research in which conflict of interest is more or less inevitable. In some ways this evolution is following the evolutionary pathways taken in research ethics over the past three or four decades. Thus, in the sixties there was a more-or-less public outcry about the unethical use of re-

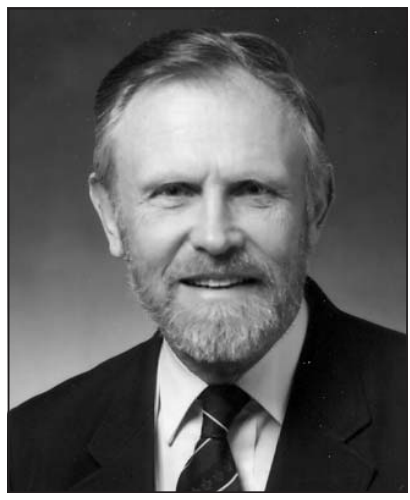
search subjects, and to this was added a number of fraudulent studies and publications, leading to a greater awareness, research guidelines, local ethics committees and mandatory statements in publications about informed consent and arm's-length peer review. Many journals now require statements about individuals' responsibility for research conduct and writing and an explicit desription of funding sources and possible conflicts of interest.

Where have we reached in this evolution? Researchers remain under great pressure to obtain industry funding, to the extent that some granting agencies will match such funding; in some areas of research the development of innovations that may be patented is an important objective. However, involvement in pharmaceutically sponsored research remains a two-edged sword; on a day-to-day basis, research is often scrutinized minutely by one of the firm's research associates, copying results and questioning procedures. Then there are research contracts that may not be minutely scrutinized during the heady flush of obtaining a grant, only to surface later when the researcher may wish to present results that are not conducive to the company's bottom line. Without belabouring the point, at the very least this can compromise scientific integrity and make the researcher's life extremely unpleasant. Journals have conflict of interest guidelines, and nowadays statements regarding funding and authorship go some way to alerting the reader. A recent Lancet editorial on the results of an industry-based study was able to point out flaws and improvements in design, and concluded that such research "has its uses" but "is insufficient on its own to establish the long term validity of current recommendations". The paper had undergone peer review, but even this is not immune from conflicts of interest, as judged from a recent wellpublicized apology in the New England Journal of Medicine. 
The Canadian Respiratory Journal up until now has not required statements regarding conflict of interest or a description of the tasks carried out by individual authors; however, where a paper is multiauthored we require all authors to sign the letter of submission and an explicit statement of financial support may be requested. Dr McFadden's concern regarding biased reporting in an industry-sponsored publication that was mailed with the Canadian Respiratory Journal has led Pulsus Group to establish new guidelines for this type of publication. First, they will be published using a format and paper that will be distinctly different from the Journal's; second, they will carry a disclaimer that the material has not been peer reviewed, or will be identified as advertising material; and third, the content will be reviewed and accepted by the Pharmaceuticals Advertising Advisory Board. The latter is an independent review agency that has a board of directors with representation from many organizations, from the Consumers' Association to the Canadian Medical Association, set up to ensure that promotional material meets scientific standards and promotes a balanced description of the various attributes of a given drug. We see these guidelines as an important first step in meeting ethical concerns regarding such publications. However, it is not yet clear what standards the board will apply, and it may not be able to peer-review the scientific quality of this type of publication, in contrast to product monographs. Thus the process may not avoid an unbalanced presentation in some respects, but the correspondence columns of the Journal are available to anyone who feels that balance needs to be restored. Furthermore, we would encourage readers to make suggestions regarding any measure that may help to maintain the Journal's scientific and ethical standards.

The pharmaceutical industry is now intimately and crucially involved in the support of university research programs, whether through shared sponsorship with government research councils, endowed academic chairs or research institutes. No one is going to suggest any diminution in such support, and their own objectives are to be respected. Lest this editorial be seen as overly negative, it also needs to be said that relations between researchers and sponsors are ususally very cordial, with much mutual trust and respect. However, continued dialogue between researchers and the industry is urgently needed, at the very least to develop guidelines or codes of conduct that are similar to the research ethics guidelines that are now well accepted, and that might formalize relationships and responsibilities while acknowledging the importance of individual scientific freedom and open discussion of research results and controversies.

Norman L Jones Editor-In-Chief Canadian Respiratory Journal 


\section{Relations dangereuses?}

$\mathrm{P}$ arfois, j'ai tellement l'impression d'être sous l'emprise des circonstances et des coïncidences qu'il ne me reste que très peu de choix, par exemple, lorsque vient le temps de rédiger un éditorial. En voici l'illustration : pendant quelques jours le mois dernier, j'ai assisté à une réunion fortement commanditée de l'Ontario Lung Association, j'ai évalué deux articles sur des essais de médicaments, j'ai pris connaissance des menaces de poursuites formulées par des sociétés pharmaceutiques contre deux chercheurs de l'Ontario, j'ai entendu parler des excuses publiques du New England Journal of Medicine au sujet des conflits d'intérêts avec les réviseurs et j'ai reçu une lettre critique de Rob McFadden, rédacteur adjoint du Journal canadien de pneumologie, à propos d'une publication commanditée, insérée dans le dernier numéro de 1999. Je suppose que toutes ces situations reflètent les liens plutôt ambivalents que nous entretenons avec l'industrie pharmaceutique qui appuie de nombreux programmes professionnels et universitaires et qui s'attend à en retirer plus que des avantages fiscaux. De nos jours, nous dépendons financièrement de l'industrie pour organiser des réunions de professionnels et d'universitaires, réunions de grande envergure qui nécessitent de grandes salles coûteuses. L'industrie est alors en droit de s'attendre à un certain rendement de ses investissements. Rien de plus évident que le congrès annuel du Collège royal à Montréal, où le nombre déplorable de participants rivalisait avec l'apathie de l'exposition pharmaceutique; le stand le plus populaire a été celui des voitures Jaguar, où l'on offrait gratuitement des essais de conduite du dernier modèle. D'autres programmes universitaires sont également redevables des fonds alloués par les sociétés pharmaceutiques pour les conférenciers, les rafraîchissements et les petites réunions, qui, dans «le bon vieux temps », semblaient faciles à organiser à partir des subventions à l'éducation locales; ce genre de financement remonte le moral, même si l'objectif pédagogique est parfois relayé au second plan. La compétition annuelle pour l'obtention de bourses de résidents et de chercheurs, qui s'est tenue l'année dernière à Sainte-Adèle au Québec (publiée dans le Journal canadien de pneumologie 2000;7; 84-94), représente un bon exemple de rassemblement où les objectifs pédagogiques et sociaux légitimes ont été largement atteints. D'autres réunions, par contre, dont celles organisées dans des régions du monde très attrayantes afin de présenter de nouveaux médicaments, semblent souscrire à des principes moins altruistes. Les articles qui y sont présentés décrivent généralement le travail qui a été mené par les sociétés pharmaceutiques plutôt que par le chercheur, qui peut utiliser un budget soufflé pour soutenir la recherche qu'il souhaite vraiment effectuer. Ces articles qui paraissent ensuite dans des publications non révisées par les pairs peuvent laisser beaucoup à désirer, comme le souligne d' ailleurs le Dr McFadden.

Les revues scientifiques semblent avoir éprouvé un

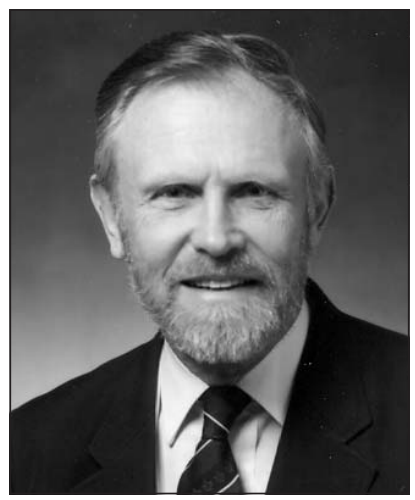
certain nombre de problèmes avec des articles de recherche commandités par des sociétés pharmaceutiques et se sont efforcées d'élaborer un protocole d'éthique dans lequel, finalement, les conflits d'intérêts sont plus ou moins inévitables. D'une certaine façon, cela fait suite au processus d'évolution de l'éthique dans le domaine de la recherche, entrepris depuis les trois ou quatre dernières décennies. Ainsi, au cours des années 60 , il y a eu un tollé plus ou moins général contre l'utilisation contraire à l'éthique des sujets de recherche, suivi d'un nombre d'études et de publications fausses qui ont mené à une sensibilisation accrue, à l'établissement de lignes de conduite en matière de recherche, à la mise sur pied de comités d'éthique ainsi qu'à la déclaration obligatoire, dans les publications, du consentement éclairé et de la révision par les pairs sans lien de dépendance. De nombreuses revues exigent maintenant des déclarations sur la responsabilité de chacun dans la conduite de la recherche et la rédaction, de même qu'une description explicite des sources de financement et des conflits d'intérêts possibles.

Où cette évolution nous a-t-elle menés? Les chercheurs subissent toujours des pressions énormes pour obtenir du financement auprès de l'industrie, au point où certains organismes subventionnaires sont prêts à investir des sommes équivalentes; dans certains domaines de recherche, la conception d'innovations susceptibles d'être brevetées constitue un objectif important. Cependant, la participation à la recherche commanditée par des sociétés pharmaceutiques demeure une épée à deux tranchants; souvent, la recherche est scrutée minutieusement par un des assistants à la recherche de la société, qui copie les résultats et pose des questions sur les protocoles utilisés. Puis, il y a les contrats de recherche qui ne sont pas nécessairement scrutés minutieusement durant la phase de la course folle au financement, mais qui le sont plus 
tard au moment où le chercheur se dit prêt à présenter les résultats mais qui ne répondent pas aux objectifs fondamentaux de la société. Sans insister davantage sur ce point, cette situation peut, à tout le moins, compromettre l'intégrité scientifique et rendre la vie d'un chercheur extrêmement désagréable. Les revues scientifiques ont élaboré des directives sur les conflits d'intérêts et, de nos jours, les déclarations sur le financement et la paternité d'une œuvre avertissent en quelque sorte le lecteur. Un éditorial récent, publié dans le Lancet, sur les résultats d'une étude commanditée par l'industrie a été en mesure de souligner les vices de conception et les améliorations à apporter, et a conclu que ce genre de recherche « avait son utilité », mais qu' elle était insuffisante en soi pour établir la validité à long terme des recommandations actuelles. L'article, bien qu'il ait été révisé par des pairs, n'était pas à l'abri des conflits d'intérêts, comme en témoignent les excuses publiées récemment dans le New England Journal of Medicine.

Jusqu'à présent, le Journal Canadien de pneumologie n'a pas exigé de déclaration sur les conflits d'intérêts ou de description de tâches effectuées par les auteurs; cependant, lorsque plusieurs auteurs participent à la rédaction d'un article, nous exigeons que tous les auteurs signent une lettre de soumission, en plus, parfois, de leur demander une déclaration explicite sur l'appui financier. Les craintes $\mathrm{du} \mathrm{D}^{\mathrm{r}} \mathrm{McFadden}$ à propos d'un reportage partial, paru dans une publication parrainée par l'industrie et inséré dans le Journal canadien de pneumologie a incité Pulsus Group à établir des nouvelles directives concernant ce genre de publications. Tout d'abord, ces articles seront publiés dans un format et sur du papier différents de ceux utilisés par la revue; ensuite, ces articles porteront un avertissement indiquant que le contenu n'a pas été révisé par des pairs ou seront présentés comme du matériel publicitaire; enfin, le contenu devra être révisé et accepté par le Conseil consultatif de publicité pharmaceutique. Celui-ci est un organisme de révision indépendant dont le conseil d'administration est composé de représentants provenant de nombreux organismes, depuis l'Association des consommateurs du Canada jusqu'à l'Association médicale canadienne. Le Conseil a été formé pour s'assurer que le matériel publicitaire respecte les normes scientifiques et diffuse une description équilibrée des divers attributs d'un médicament donné. Nous considérons ces directives comme une première étape importante pour apaiser les craintes d'ordre éthique concernant ce genre de publication. Toutefois, il n'est clair quelles normes appliquera le Conseil et il peut ne pas être en mesure de soumettre à l'analyse des pairs la qualité scientifique de ce genre de publication, contrairement aux monographies. Aussi, peut-être ne sera-t-il pas possible d'éviter une présentation déséquilibrée à certains égards, mais les colonnes de la revue réservées aux lecteurs sont à la disposition de quiconque juge nécessaire de rétablir les faits. De plus, nous encourageons les lecteurs à faire des suggestions sur toute mesure qui pourrait aider la revue à maintenir les normes scientifiques et éthiques.

L'industrie pharmaceutique participe très activement à l'appui financier des programmes de recherche universitaire, que ce soit par la voie de partenariats établis avec des conseils de recherche gouvernementaux, de chaires fondées ou d'instituts de recherche. Nul n'aura l'idée de suggérer une diminution de l'appui financier, et les objectifs des bailleurs de fonds doivent être respectés. Pour faire contrepoids au ton de l'éditorial qui pourrait laisser une impression trop négative, je tiens à préciser que les relations entre les chercheurs et les commanditaires sont, en général, très cordiales et qu'elles se font dans un esprit de confiance et de respect mutuels. Cependant, il est nécessaire d'entretenir le dialogue entre les chercheurs et l'industrie, tout au moins pour élaborer des directives ou des codes de conduite semblables à ceux qui sont en vigueur dans la recherche et qui sont maintenant bien acceptés. Le maintien du dialogue pourrait donner un caractère officiel aux relations et aux responsabilités de chacun, tout en reconnaissant l'importance de la liberté individuelle et de la discussion ouverte sur les résultats de la recherche et les controverses.

Norman L Jones

Rédacteur en chef Journal Canadien de pneumologie 


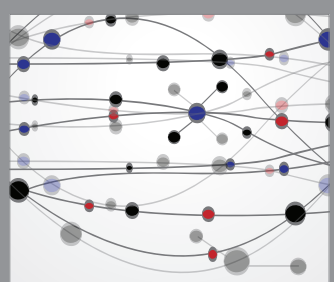

The Scientific World Journal
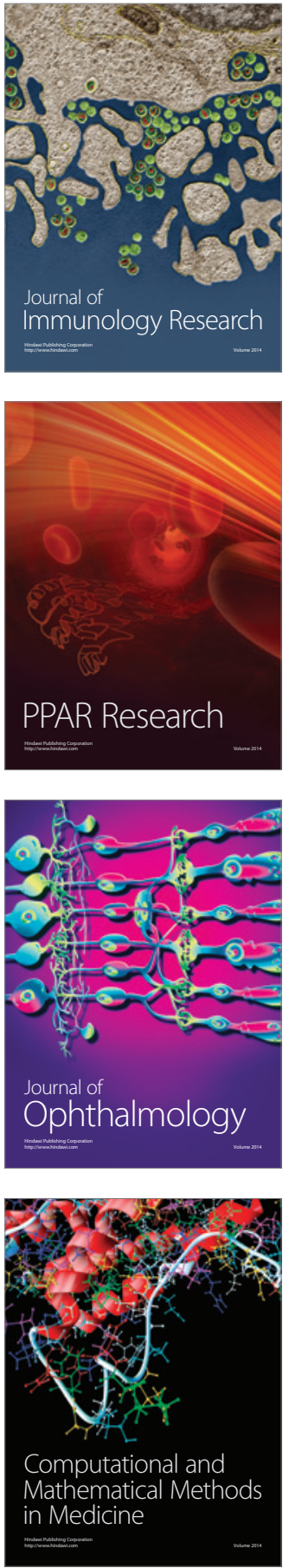

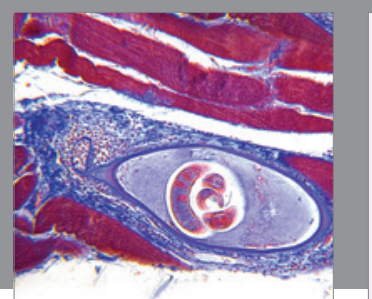

Gastroenterology Research and Practice

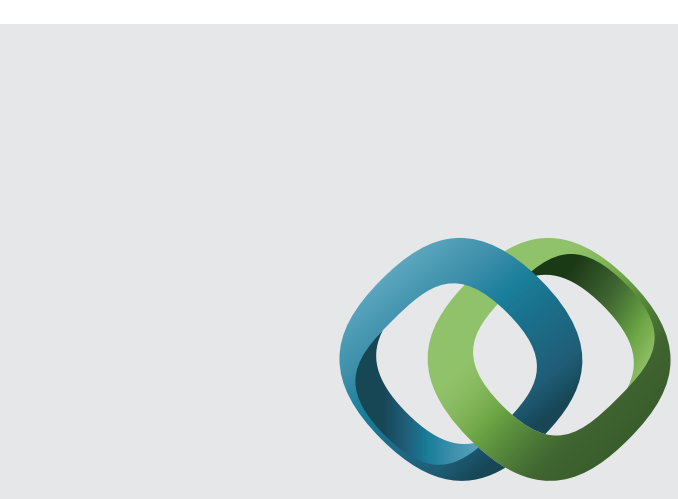

\section{Hindawi}

Submit your manuscripts at

http://www.hindawi.com
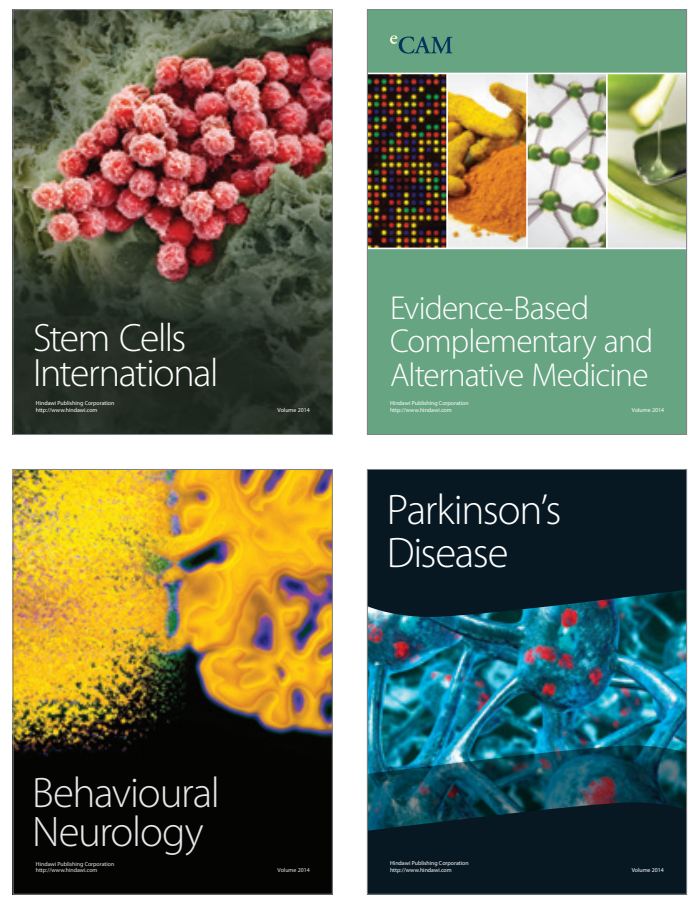
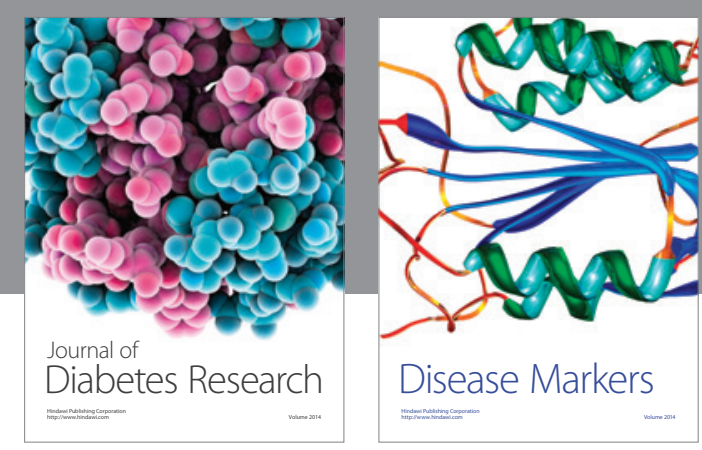

Disease Markers
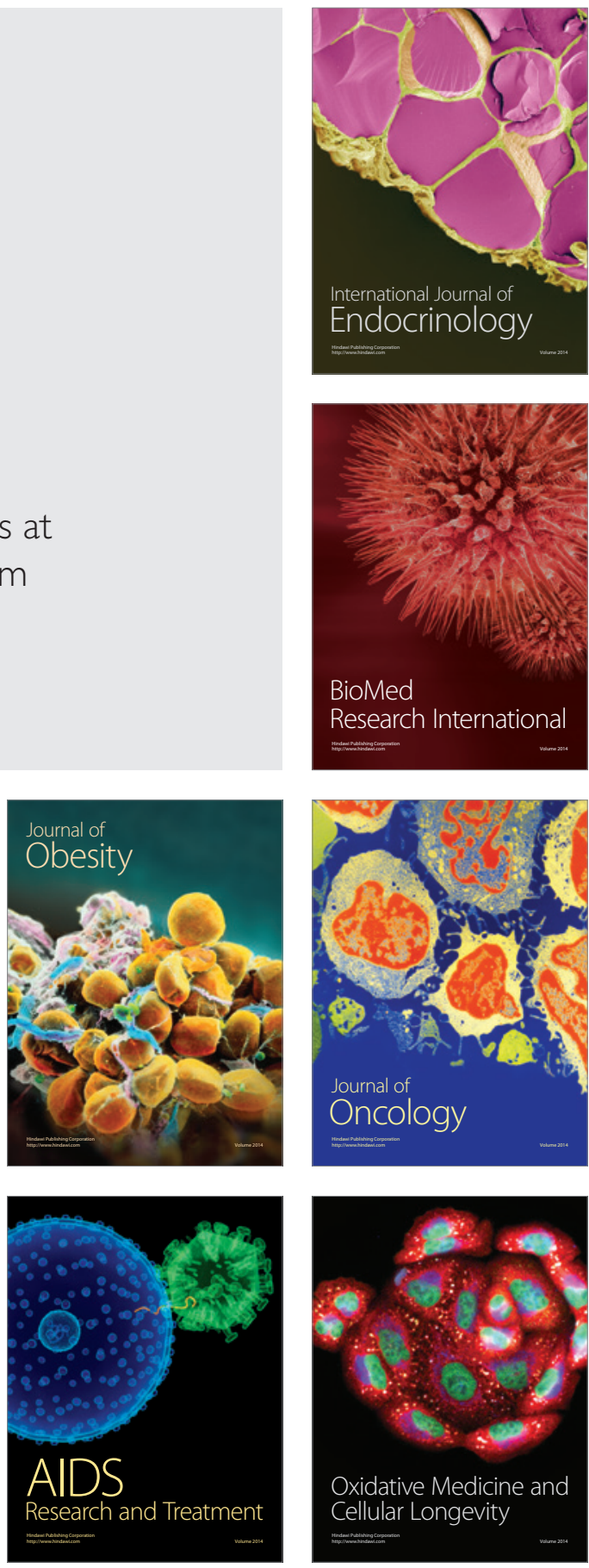